Review

\title{
Inflammatory Reactions in the Pathogenesis of Atherosclerosis
}

\author{
Jianglin Fan ${ }^{1}$ and Teruo Watanabe ${ }^{2}$ \\ ${ }^{1}$ Laboratory of Cardiovascular Disease, Institute of Basic Medical Sciences, University of Tsukuba, Tsukuba, Japan \\ ${ }^{2}$ Saga Medical School, Saga, Japan.
}

\begin{abstract}
Atherosclerosis and its complications constitute the most common causes of death in Western societies and Japan. Although several theories or hypotheses about atherogenesis have been proposed during the past decades, none can completely explain the whole process of the pathogenesis of atherosclerosis because this disease is associated with multiple risk factors. In spite of this, the concept that atherosclerosis is a specific form of chronic inflammatory process resulting from interactions between plasma lipoproteins, cellular components (monocyte/macrophages, T lymphocytes, endothelial cells and smooth muscle cells) and the extracellular matrix of the arterial wall, is now well accepted. Histologically, atherosclerotic lesions from the early-stage (fatty streak) to more complicated lesions possess all the features of chronic inflammation. It has been demonstrated that atherogenic lipoproteins such as oxidized low density lipoprotein (LDL), remnant lipoprotein ( $\beta$ - VLDL) and lipoprotein [Lp] (a) play a critical role in the pro-inflammatory reaction, whereas high density lipoprotein (HDL), anti-atherogenic lipoproteins, exert anti-inflammatory functions. In cholesterol-fed animals, the earliest events in the arterial wall during atherogenesis are the adhesion of monocytes and lymphocytes to endothelial cells followed by the migration of these cells into the intima. It has been shown that these early events in atherosclerosis are triggered by the presence of high levels of atherogenic lipoproteins in the plasma and are mediated by inflammatory factors such as adhesion molecules and cytokines in the arterial wall. The development of genetically modified laboratory animals (transgenic and knock-out mice and transgenic rabbits) has provided a powerful approach for dissecting individual candidate genes and studying their cause-and-effect relationships in lesion formation and progression. The purpose of this article is to review the recent progress regarding the inflammatory processes during the development of atherosclerosis based on both human and experimental studies. In particular, we will address the mechanisms of atherogenic lipoproteins in terms of inflammatory reactions associated with hypercholesterolemia. Understanding the molecular mechanisms responsible for inflammatory reactions during atherogenesis may help us to develop novel therapeutic strategies to control, treat and prevent atherosclerosis in the future. J Atheroscler Thromb, 2003; 10: 63-71.
\end{abstract}

Keywords: Atherosclerosis, Inflammation, Hypercholesterolemia, Lipoprotein (a), Foam cells, Plaque rupture

\section{Early stage of atherosclerosis}

The accumulation of lipid-loaded cells underlying the endothelium of large arteries, namely, fatty streaks or dots, is a hallmark of early-stage atherosclerotic lesions (Fig. 1).

Address for correspondence: Teruo Watanabe, Vice President, Saga Medical School, 5-1-1 Nabeshima, Saga, Saga 849-8501, Japan. E-mail: nabeteru@post.saga-med.ac.jp or J-LFAN@md. Tsukuba. ac. jp Received December 27, 2002.

Accepted for publication January 6, 2003.
Numerous studies of either human atherosclerotic lesions or cholesterol-fed animals have shown that these lipidloaded cells mainly originate from blood-born monocytes subsequently differentiated into macrophages (1). These macrophages engulf a large amount of lipids deposited in the subintimal space and take on the appearance of foamy structures, designated as foam cells. It has become clear that a number of scavenger receptors on the cellular surface are involved in the lipid [mainly lipids derived from oxidized low density lipoprotein (oxLDL)] influx into macrophages $(2,3)$. Three major lipoproteins are often 

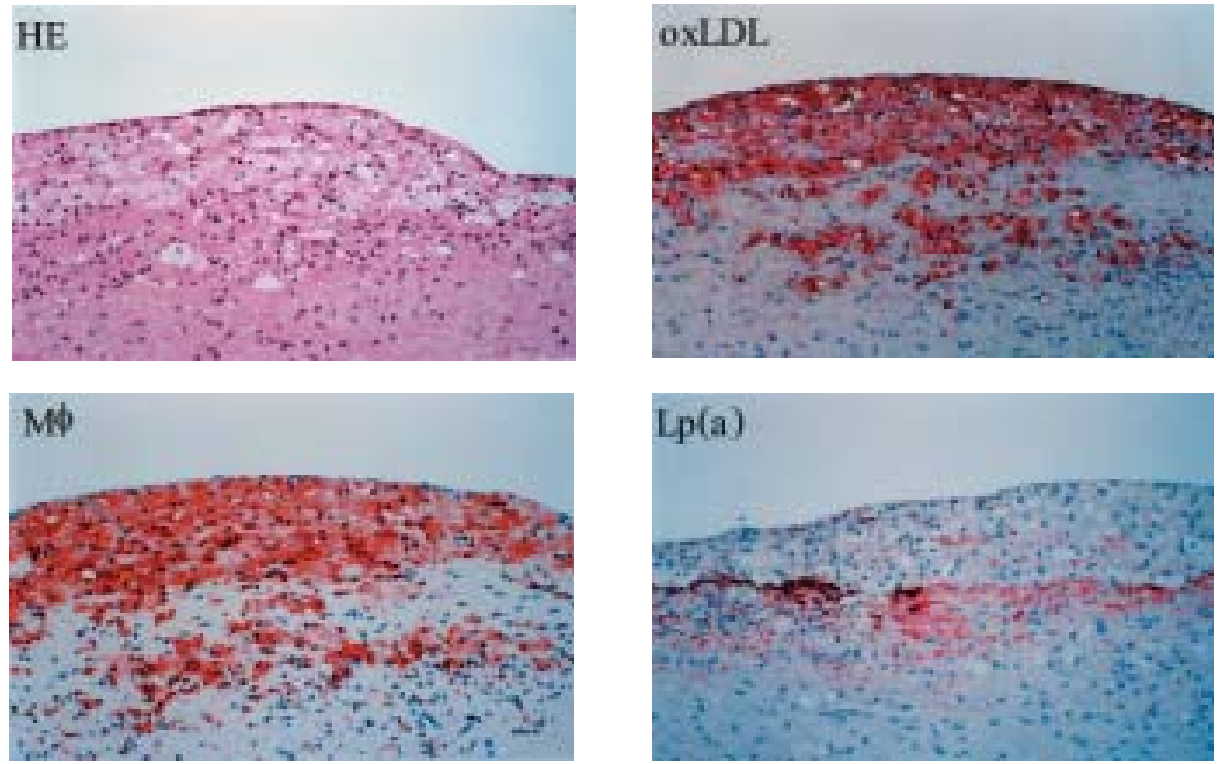

Fig. 1. A typical fatty streak seen in human atherosclerosis. The lesion is composed of foam cells with clear cytoplasm. The lesion is stained with three monoclonal antibodies for monocytes (HAM), oxidized LDL (FOH1a/DLH3), and apo (a). These foam cells are derived from macrophages as they are stained by the HAM antibody. Oxidized LDLs, the most atherogenic lipoproteins, are closely associated with foam cells. On the other hand, Lp (a), another atherogenic lipoprotein, is basically deposited around the extracellular matrix.

observed in the lesions and are considered to be atherogenic when elevated in the plasma: they include low density lipoprotein (LDL); especially small dense LDL, remnant lipoproteins (also known as $\beta-\mathrm{VLDL}$ found in both type III hyperlipidemia and cholesterol-fed animals) and lipoprotein (a) $[\mathrm{Lp}(\mathrm{a})]$ (4). These atherogenic lipoproteins, once deposited in the intima, are subjected to chemical modifications, such as oxidation, thereby leading to a series of biological reactions. Compared to oxLDL, which is invariably associated with foam cells in the lesions, LP (a), although also present in the lesions, is seldom, if ever, associated with foam cell formation. $L p(a)$ is inclinedand it tends to be associated with the extracellular matrix (Fig. 1).

The biological functions of $L p(a)$ and whether it plays a role in lesion development remain unknown, but recent studies in our laboratory using human apolipoprotein (a) [apo (a)] transgenic rabbits have revealed that $L p(a)$ may act as a pro-inflammatory mediator that augments the lesion formation $(5,6)$. In addition to the foam cells in the lesions, T lymphocytes are scattered around macrophages and foam cells (Fig. 2) $(7,8)$. Electron microscopic studies of human atherosclerotic lesions have revealed that there is an intimate interaction between T cells and macrophages and that the $T$ cells involved in this interaction are usually in the activated state as they express major histocompatibility complex (MHC) class II antigen. We demonstrated that in hypercholesterolemic rats, T cells actually predominate in the incipient phase (cholesterol-rich diet
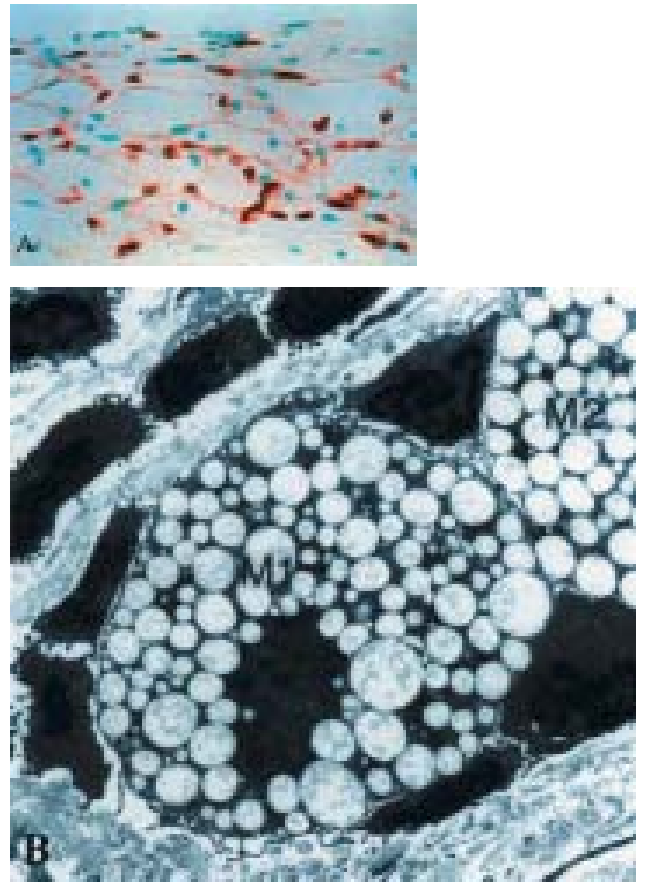

Fig. 2. Demonstration of T lymphocytes in human atherosclerotic lesions. T lymphocytes (CD8 positive) are found around foam cells, as shown by immunohistochemical staining with OKT8 antibody (A). Electron microscopic observation shows that a foam cell is surrounded by five lymphocytes in a rosette pattern and an intimate cellular interaction between foam cells and lymphocytes and between lymphocytes (B). 
for 2 weeks) of the lesion formation and are gradually surpassed by monocyte infiltration in later stages $(9,10)$. Therefore, one can envision that these T cells are not simply bystanders in the lesions, but instead actively participate in the lesion progression, possibly through the release of diverse cytokines $(9,11,12)$. Immumohistochemical studies also showed that a number of cytokines derived from different vascular cells are present in lesions at all stages (11). One of the most notable cytokines derived from $T$ lymphocytes is interferon gamma (INF- $\gamma$ ), which has been shown to play diverse roles in mediating foam cell formation, the proliferation of smooth muscle cells and regulating the production of matrix metalloproteinases, thereby influencing plaque stability (see below). Taken together, these observations suggest that cell-mediated immunity mechanisms may be involved in the pathogenesis of atherosclerosis.

\section{Mechanisms for the initiation and progression of atherosclerosis}

Cholesterol-fed animals have been widely used for investigating the early events during atherogenesis, which cannot usually be studied in humans since the lesions develop over a period of several decades. A number of pioneer studies, including ours own, during the1980s using cholesterol-fed animals, revealed that among the earliest events in the arterial wall of choleterol-fed animals is the adherence of mononuclear cells to endothelial cells, as observed by electron microscopy and immunohistochemistry (13) (Fig. 3). Later, it was found that mononuclear cell adherence is triggered by a number of adhesion molecules on endothelial cells, such as vascular cell adhesion molecule-1 (VCAM-1), intracellular adhesion molecule-1 (ICAM-1), P-secletin and E-secletin (14-19). Increased expression of these molecules is considered to be responsible for the adherence to endothelial cells of monocytes and $\mathrm{T}$ lymphocytes and for their preferential binding to the arterial surface (20). These adhesion molecules are highly up-regulated by the elevation of the levels of atherogenic lipoproteins [oxLDL and Lp (a)] and cytokines in vitro (15). Blocking these molecules by injecting specific antibodies against them leads to a remarkable reduction of intimal cells in hypercholesterolemic animals (16). Direct evidence to support the notion that these molecules are critical in the initiation of the lesion formation has been derived from studies of several genetically modified mice in which these molecules are genetically deficient $(19,21)$. Cybulsky and coworkers generated VCAM-14D/4D knock-out (KO) mice and showed by studying them in comparison to ICAM-1 deficient mice that VCAM plays a more important role than ICAM-1 in initiating the monocyte adherence to endothelial cells during atherogenesis (21).

After monocytes and T lymphocytes bind to the surface of the arterial wall, they migrate into the subendothelial

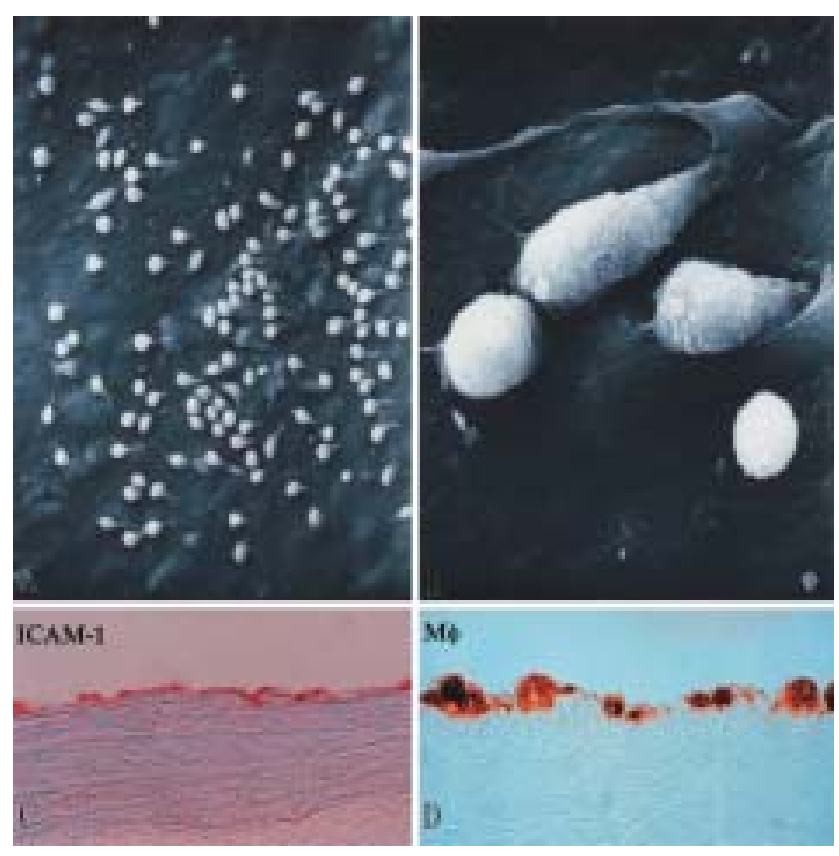

Fig. 3. The earliest events of arterial intima in cholesterol-fed animals. Rats were fed a cholesterol-rich diet for 4 weeks and their aortas were studied by scanning electron microscopy and immunohistochemical staining. A number of leukocytes adhere to the surface of the endothelial cell surface (A) and some are in the process of entering the subendothelial space (B). Immunohistochemical staining reveals that endothelial cells express increased levels of an adhesion molecule, ICAM-1(C) and that the accumulated intimal cells are macrophages (D).

space, where they then differentiate and are transformed into macrophages and foam cells. This subendothelial migration is induced by the presence of bioreactive mediators called chemoattractants in the intima. Several candidate chemoattractants have been documented as functional in this respect in vitro; all of these candidates must satisfy two requirements: they should be present in the intima and should act specifically as chemoattractants for monocytes or lymphocytes. During the past 20 years, a number of chemoattractants have been shown to be capable of inducing monocyte chemotaxis: they include oxLDL, Lp (a), cytokines [ monocyte chemoattractant protein (MCP-1), interleukin-1 (IL-1) and tumor necrosis factor-alpha (TNF $\alpha$ )] and degraded collagens and elastins (22). Among all the mediators reported thus far, MCP-1 and Lyso-PC (a component of oxLDL) may be the most important and are the best-characterized chemoattractants in the lesions (23). MCP-1 has been found in the lesions in the early stage and can be produced by endothelial cells and also macrophages themselves (24). The function of MCP-1 depends on the specific receptor CCR-2 present on the surface of monocytes. Deficiency in either MCP-1 protein or its receptor CCR-2 significantly reduces the le- 
sion development in apoE KO mice $(25,26)$, suggesting that MCP-1 is a critical mediator of the recruitment of monocytes in the intima. This notion is further strengthened by the finding that CCR-2-deficient mice show increased susceptibility to tuberculosis, another type of chronic inflammation (27). More recently, using a specific CCR-2 receptor inhibitor, Yamashita et al. showed that administration of propagermanium to apoE-deficient mice reduces atherosclerosis by inhibiting macrophage infiltration (28). In addition to acting as a chemoattractant for monocytes, oxLDL can inhibit macrophage mobility (29). Although the mechanism of this phenomenon has not been fully clarified, such a dual function of oxLDL in terms of directing cell mobility may help to explain why macrophages or foam cells failed to return the circulation as they are supposed to do. These findings suggest that in future experiments we should test whether we can treat patients with coronary heart disease (CHD) or prevent atherosclerosis using drugs that can target either adhesion molecules or chemoattactants for monocyte/macrophages. Figure 4 summarizes the postulated mechanisms for the pathogenesis of atheroscclerosis, with emphasis on inflammatory reactions.

\section{Plaque stability and inflammatory reactions}

Typical atheroclerotic plaque (also called atheroma or fibrous plaque) contains a lipid or necrotic core covered by a layer of fibrotic cap consisting of a mixture of smooth muscle cells and extracellular matrix (Fig. 5). The base of the lesion, called the "shoulder," often contains a number of macrophage-derived foam cells and T lymphocytes. It is generally believed that these components can determine the fate of the plaque, so-called plaque stability and destability. Basically, two kinds of common plaque can be morphologically differentiated and are clinically relevant: stable plaque and unstable or vulnerable plaque (Fig. 5). Stable plaque is usually composed of a small lipid core and covered by a thick fibromuscular cap with more smooth muscle cells and extracellular matrix. Unstable plaque often contains a large pool of lipid core, and a thin cap and a large number of inflammatory cells, especially at the shoulder, may be present. Morphologically speaking, stable plaques are the major risks for

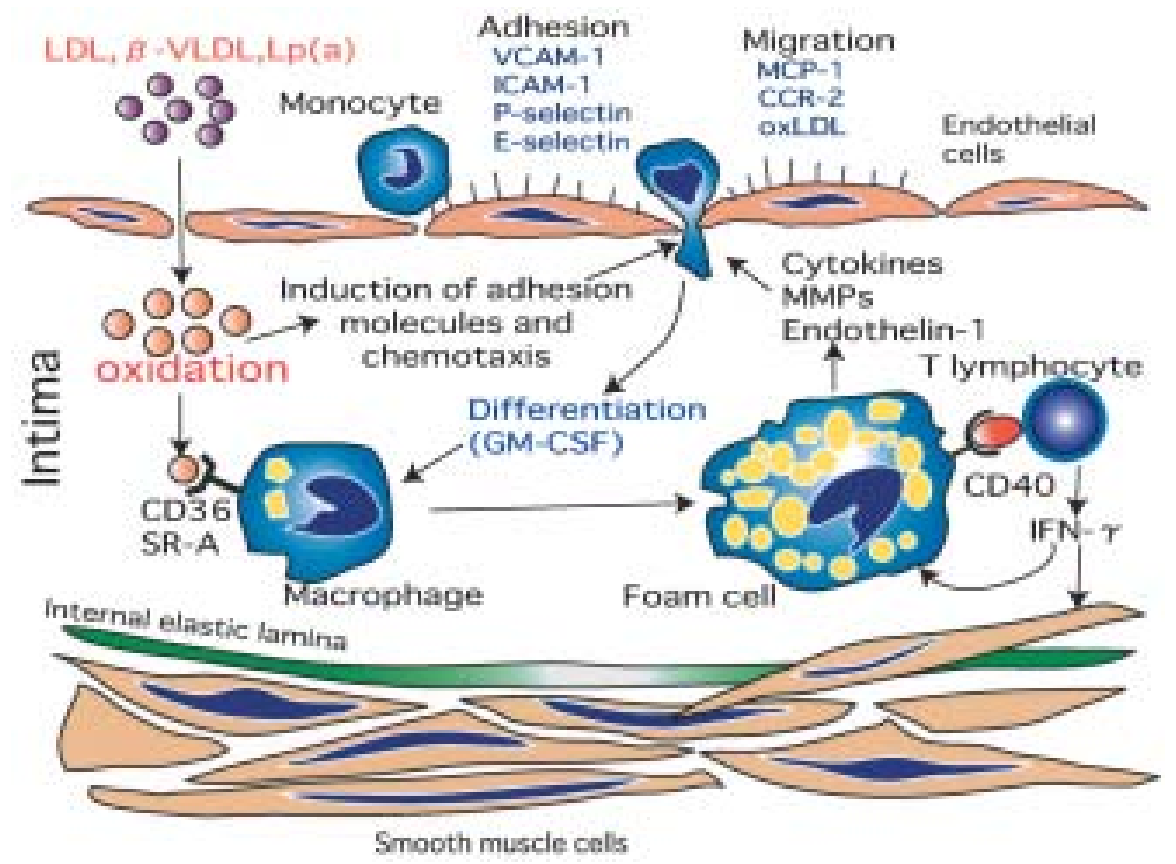

Fig. 4. Postulated mechanisms of the pathogenesis of atherosclerosis. The elevation of plasma atherogenic lipoproteins [LDL, beta-VLDL and Lp (a)] may lead to the deposition of these lipoproteins in the intima. These atherogenic lipoproteins may induce a series of biological changes, including increased adhesion molecules in endothelial cells, and the adhesion and migration of monocytes and T lymphocytes. Monocytes may differentiate into macrophages under the action of GMCSF. Macrophages may take up deposited atherogenic lipoproteins through scavenger receptors and be transformed into foam cells. Close interactions among foam cells, T lymphocytes and smooth muscle cells together with diverse cytokines and other biological effects may take place, ultimately deciding the fate of the lesions. 
stenosis or occlusion when it causes a significant reduction of the vascular lumen. Vulnerable plaques, however, is fatal and the outcome of its formation is ominous, because regardless of its size, a vulnerable plaque usually leads to acute coronary syndrome if it ruptures or is associated with thrombosis. A representative unstable vulnerable plaque of coronary atherosclerosis is shown in Fig. 6. The mechanism (s) of plaque rupture is not fully understood, but the accumulation of macrophages and $T$ lymphocytes may play a critical role in this regard because macrophages can produce matrix metalloproteinases (MMPs), which may destruct the thin cap and potentiate the rupture (30-32). Several cytokines in the lesions may also upregulate the secretion of MMPs such as TNF, IL-1 and MG-CSF (33). In addition, an intimate interaction between $T$ lymphocytes and macrophages may also regulate the production of macrophage-derived MMPs. The CD-40 ligand on T cells can bind to the CD-40 receptor on macrophages, a process which has been shown to induce MMP synthesis (34). T lymphocytes can also influence plaque stability through the release of INF- $\gamma$, which inhibits smooth muscle cell proliferation and matrix production. In the future, it will be important to test whether the use of MMP inhibitors can reduce the risk of plaque rupture in CHD patients.

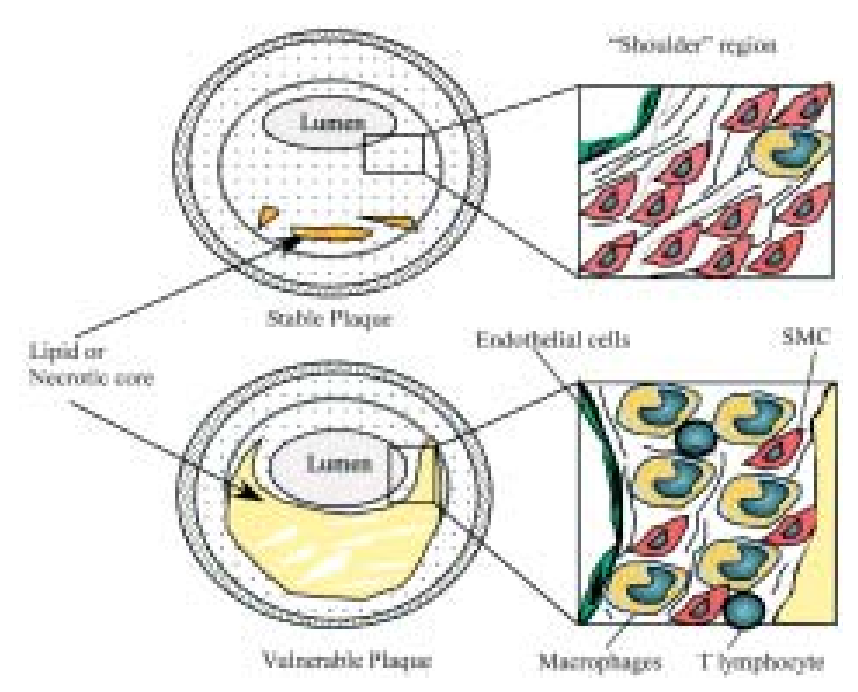

Fig. 5. Schematic illustration of stable and unstable plaques. Stable plaque usually has a well-preserved lumen and a thick fibromuscular cap in which there are numerous synthetic-type smooth muscle cells with abundant extracellular matrix. Lipid cores are almost always small. Vulnerable plaque usually has a large lipid or necrotic core and a thin fibrotic cap beneath which there are numerous macrophages together with $\mathrm{T}$ lymphocytes and smooth muscle cells.

\section{Lipoproteins and inflammation}

Since atherosclerosis is a specific type of chronic inflammatory process, as discussed above, it is important to identify the inflammatory factors involved in atherosclerosis (35). The causal relationship between blood cholesterol and atherosclerosis is no longer in doubt, therefore, we should know which cholesterol or lipoprotein is bad or atherogenic (35). All atherogenic lipoproteins, once deposited in the intima, may exert direct or indirect proinflammatory effects. Table 1 summarizes the potential roles of oxLDL in atherogenesis, with special reference to its properties as an inflammatory mediator. As mentioned above, oxLDL can induce adhesion molecule expression on endothelial cells and trigger the migration of monocytes towards the intima. On the other hand, oxLDL can stimulate the production of many inflammatory mediators (e. g. endothelin-1) from other vascular cells, in turn resulting in diverse inflammatory responses in the arterial wall $(36,37)$. Using apoE KO mice, our laboratory demonstrated that the chronic administration of endothelin-1 A/B receptor antagonist SB209670 results in a significant reduction of atherosclerosis, independent of the plasma levels of cholesterol (37). Therefore, it is likely that, similar to lipid-lowering drugs (such as statins),

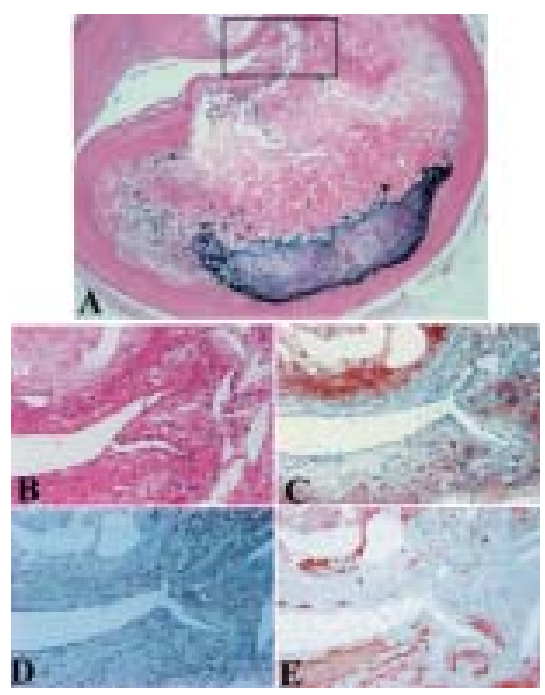

Fig. 6. A typical unstable plaque of human coronary atherosclerosis. The lesion is stained with antibodies HAM for monocytes, HF35 for smooth muscle $\alpha$-actin and apo (a), The lesion contains a thin cap beneath which a number of macrophages ( $B$ and $C$ ) are present. In the cap of the lesion, there are almost no smooth muscle cells (D). Presumably, macrophage-derived MMPs may reduce plaque stability and cause a rupture. It is notable that $L p(a)$ is also present in the lesion but is apparently not associated with macrophages (E). 
Table 1. Possible proatherogenic effects of oxidized LDL

\begin{tabular}{ll}
\hline Effects & Possible mechanisms \\
\hline Adhesion of monocytes to endothelial cells $\uparrow$ & Increased expression of adhesion molecules on endothelial cells \\
Monocyte and T lymphocyte chemotaxis $\uparrow$ & Induction of MCP-1 production and direct chemoattractant effect \\
Scavenger receptor A and CD36 $\uparrow$ & Activation of AP-1 and ets transcription factors \\
Foam cell formation $\uparrow$ & Enhanced uptake of oxLDL mediated by scavenger receptor \\
Induction of proinflammatory genes & Activation of NFאB, and AP-1, and increased cAMP \\
Increased cellular death & Activation of apoptosis and formation of cholesterol crystals \\
Thrombosis $\uparrow$ & Induction of tissue factor, increased platelet aggregation \\
Impaired vascular functions & Dysfuction of ET-1 and NO \\
Plaque rupture $\uparrow$ & Increased MMPs production \\
\hline
\end{tabular}

$\uparrow:$ enhanced

Table 2. Possible anti-atherogenic effects of HDL

\begin{tabular}{ll}
\hline Effects & Possible mechanisms \\
\hline Inhibition of lesion formation $\downarrow$ & Reverse cholesterol transport \\
Adhesion of monocytes to endothelial cells $\downarrow$ & Inhibition of adhesion molecules and decreased cytokine production \\
Thrombosis $\downarrow$ & Modulation of endothelial antithrombotic and profibrinolytic properties \\
Antioxidant effects $\uparrow$ & Modulation of paraoxonase \\
Cellular death $\downarrow$ & Inhibition of apoptosis \\
Cellular proliferation $\downarrow$ & Inhibition of SMC proliferation \\
\hline
\end{tabular}

$\downarrow$ : reduced or inhibited

anti-inflammatory agents may be useful for the treatment of atherosclerosis in the future. In addition to oxLDL, Lp (a), another atherogenic lipoprotein, may also lead to an inflammatory process by inducing the expression of adhesion molecules on endothelial cells, the chemotaxis of monocytes, and the proliferation of smooth muscle cells (38). In one of our recent studies using transgenic rabbits expressing human apo (a), we demonstrated that $L p(a)$ in transgenic rabbits which do not have endogenous apo (a) showed increased aortic atherosclerosis in comparison with control rabbits. Atherosclerotic lesions in transgenic rabbits are characterized by increased cellular proliferation, and $L p(a)$ is often deposited in the lesions. We initially hypothesized that $L p($ a) may stimulate smooth muscle cell dedifferentiation in addition to proliferation and found that a large number of intimal cells are positively stained with SMemb, a monoclonal antibody against the smooth muscle myosin heavy chain isoform (38). In addition, $L p(a)$ has the ability to inhibit fibrinolytic activity by increasing plasma plasminogen activator-1 production (38). More recently, we demonstrated that $L p(a)$ enhances advanced atherosclerosis and vascular calcification in WHHL transgenic rabbits (39). These results again suggest that atherogenic lipoproteins exert diverse effects by inducing chronic inflammatory reactions during lesion formation. Importantly, these atherogenic lipoproteins can augment the production of cytokines by vascular cells, and through the autocrine and paracrine mechanisms, the inflammatory reaction may lead to a vicious cycle resulting in lesion progression. In contrast, HDL, an anti-atherogenic lipoprotein that protects against atherogenesis via reverse cholesterol transport, plays an important role as an anti-inflammatory factor. Several beneficial functions of HDL have been documented (Table 2) and it is expected that the therapeutic use of HDL elevation may open avenues for the treatment of atherosclerosis in the future (40).

\section{Perspectives}

Vascular biology research has progressed remarkably in the last decade, resulting in a better understanding of vascular cell responses to inflammatory stimuli. The role of vascular cells and dyslipidemias is critical during inflammation and is of particular importance in atherosclerosis. It is clear that most vascular inflammatory responses are mediated through the nuclear factor $\kappa \mathrm{B}$ system. In addition, PPARs (steroid hormone nuclear receptors) have been shown to act as ligand-activated transcription factors controlling the expression of a number of genes in the vascular wall (41-44). Understanding the molecular mechanisms of these phenomena may not only provide insights into the pathogenesis of atherosclerosis but may also help us to develop new therapeutic strategies to treat atherosclerosis in the future. In this respect, it will be important to determine how to control inflammatory pro- 
cesses during the lesion formation and progression and whether anti-inflammatory agents are clinically beneficial. Macrophage foam cells in the lesions may be a target for therapeutic intervention in the future (45). At present, treating patients with hypercholesterolemia is still the primary clinical task, but inhibiting the inflammatory process using various anti-inflammatory antagonists may help to prevent plaque rupture and reduce myocardial infarction. It can be anticipated that in the next few years, new antiinflammatory agents may be developed as therapeutic tools as the molecular mechanisms of atherosclerosis and dyslipidemias are clarified.

Acknowledgements: This work was supported by Grantsin-Aid for Scientific Research from the Ministry of Education, Science, and Culture of Japan, the Japan Society for the Promotion of Sciences (JSPS-RFTF96I00202) and TARA project of the University of Tsukuba.

\section{References}

(1) Watanabe T, Tokunaga O, Fan JL and Shimokama T: Atherosclerosis and macrophages. Acta Pathol Jpn, 39: 473-486, 1989

(2) Febbraio M, Hajjar DP and Silverstein RL: CD36: a class B scavenger receptor involved in angiogenesis, atherosclerosis, inflammation, and lipid metabolism. J Clin Invest, 108: 785-791, 2001

(3) Boullier A, Bird DA, Chang MK, Dennis EA, Friedman P, Gillotre-Taylor K, Horkko S, Palinski W, Quehenberger O, Shaw P, Steinberg D, Terpstra V and Witztum JL: Scavenger receptors, oxidized LDL, and atherosclerosis. Ann NY Acad Sci, 947: 21422; discussion 222-223, 2001

(4) Glass CK and Witztum JL: Atherosclerosis. the road ahead. Cell, 104: 503-516, 2001

(5) Ichikawa $T$, Unoki $H$, Sun $H$, Shimoyamada $H$, Marcovina S, Shikama $\mathrm{H}$, Watanabe $\mathrm{T}$ and Fan $\mathrm{J}$ : Lipoprotein (a) promotes smooth muscle cell proliferation and dedifferentiation in atherosclerotic lesions of human apo (a) transgenic rabbits. Am J Pathol, 160: 227-236, 2002

(6) Fan J, Shimoyamada H, Sun H, Marcovina S, Honda $\mathrm{K}$ and Watanabe $\mathrm{T}$ : Transgenic rabbits expressing human apolipoprotein (a) develop more extensive atherosclerotic lesions in response to a cholesterolrich diet. Arterioscler Thromb Vasc Biol, 21: 88-94, 2001

( 7 ) Shimokama T, Haraoka S and Watanabe T: Immunohistochemical and ultrastructural demonstration of the lymphocyte-macrophage interaction in human aortic intima. Mod Pathol, 4: 101-107, 1991

( 8 ) Shimokama T, Haraoka S and Watanabe T: Participation of T lymphocytes and macrophages in atherogenesis. J Atheroscler Thromb, 1: S14-S20, 1994
( 9 ) Haraoka S, Shimokama T and Watanabe T: Participation of T lymphocytes in atherogenesis: sequential and quantitative observation of aortic lesions of rats with diet-induced hypercholesterolaemia using en face double immunostaining. Virchows Arch, 426: 307-315, 1995

(10) Haraoka S, Shimokama T and Watanabe T: Role of T lymphocytes in the pathogenesis of atherosclerosis: animal studies using athymic nude rats. Ann NY Acad Sci, 811: 515-518, 1997

(11) Kishikawa H, Shimokama $T$ and Watanabe T: Localization of $\mathrm{T}$ lymphocytes and macrophages expressing IL-1, IL-2 receptor, IL-6 and TNF in human aortic intima. Role of cell-mediated immunity in human atherogenesis. Virchows Arch A Pathol Anat Histopathol, 423: 433-442, 1993

(12) Hansson GK: Regulation of immune mechanisms in atherosclerosis. Ann NY Acad Sci, 947: 157-65; discussion 165-166, 2001

(13) Watanabe T, Hirata M, Yoshikawa Y, Nagafuchi $Y$ and Toyoshima $\mathrm{H}$ : Role of macrophages in atherosclerosis. Sequential observations of cholesterol-induced rabbit aortic lesion by the immunoperoxidase technique using monoclonal antimacrophage antibody. Lab Invest, 53: 80-90, 1985

(14) Cybulsky MI and Gimbrone MA, Jr: Endothelial expression of a mononuclear leukocyte adhesion molecule during atherogenesis. Science, 251: 788-791, 1991

(15) Kume N, Cybulsky MI and Gimbrone MA, Jr: Lysophosphatidylcholine, a component of atherogenic lipoproteins, induces mononuclear leukocyte adhesion molecules in cultured human and rabbit arterial endothelial cells. J Clin Invest, 90: 1138-1144, 1992

(16) Nie Q, Fan J, Haraoka S, Shimokama T and Watanabe T: Inhibition of mononuclear cell recruitment in aortic intima by treatment with anti-ICAM-1 and anti-LFA-1 monoclonal antibodies in hypercholesterolemic rats: implications of the ICAM-1 and LFA-1 pathway in atherogenesis. Lab Invest, 77: 469-482, 1997

(17) Dong ZM, Chapman SM, Brown AA, Frenette PS, Hynes RO and Wagner DD: The combined role of $\mathrm{P}-$ and $\mathrm{E}-$ selectins in atherosclerosis. J Clin Invest, 102: 145-152, 1998

(18) Nakashima Y, Raines EW, Plump AS, Breslow JL and Ross R: Upregulation of VCAM-1 and ICAM-1 at atherosclerosis-prone sites on the endothelium in the ApoE-deficient mouse. Arterioscler Thromb Vasc Biol, 18: 842-851, 1998

(19) Collins RG, Velji R, Guevara NV, Hicks MJ, Chan L and Beaudet AL: P-Selectin or intercellular adhesion molecule (ICAM) -1 deficiency substantially protects against atherosclerosis in apolipoprotein E-de- 
ficient mice. J Exp Med, 191: 189-194, 2000

(20) Watanabe T and Fan J: Atherosclerosis and inflammation mononuclear cell recruitment and adhesion molecules with reference to the implication of ICAM1/LFA-1 pathway in atherogenesis. Int J Cardiol, 66: S45-S55, 1998

(21) Cybulsky MI, liyama K, Li H, Zhu S, Chen M, liyama M, Davis V, Gutierrez-Ramos JC, Connelly PW and Milstone DS: A major role for VCAM-1, but not ICAM-1, in early atherosclerosis. J Clin Invest, 107: 1255-1262, 2001

(22) Ross R: Atherosclerosis an inflammatory disease. N Engl J Med, 340: 115-126, 1999

(23) McMurray HF, Parthasarathy S and Steinberg D: Oxidatively modified low density lipoprotein is a chemoattractant for human T lymphocytes. J Clin Invest, 92: 1004-1008, 1993

(24) Takeya M, Yoshimura T, Leonard EJ and Takahashi $\mathrm{K}$ : Detection of monocyte chemoattractant protein1 in human atherosclerotic lesions by an anti-monocyte chemoattractant protein-1 monoclonal antibody. Hum Pathol, 24: 534-539, 1993

(25) Boring L, Gosling J, Cleary M and Charo IF: Decreased lesion formation in CCR2-/-mice reveals a role for chemokines in the initiation of atherosclerosis. Nature, 394: 894-897, 1998

(26) Gosling J, Slaymaker S, Gu L, Tseng S, Zlot CH, Young SG, Rollins BJ and Charo IF: MCP-1 deficiency reduces susceptibility to atherosclerosis in mice that overexpress human apolipoprotein B. J Clin Invest, 103: 773-778, 1999

(27) Peters W, Scott HM, Chambers HF, Flynn JL, Charo IF and Ernst JD: Chemokine receptor 2 serves an early and essential role in resistance to Mycobacterium tuberculosis. Proc Natl Acad Sci USA, 98: 79587963, 2001

(28) Yamashita T, Kawashima S, Ozaki M, Namiki M, Inoue N, Hirata K and Yokoyama M: Propagermanium reduces atherosclerosis in apolipoprotein E knockout mice via inhibition of macrophage infiltration. Arterioscler Thromb Vasc Biol, 22: 969-974, 2002

(29) Quinn MT, Parthasarathy S, Fong LG and Steinberg D: Oxidatively modified low density lipoproteins: a potential role in recruitment and retention of monocyte/macrophages during atherogenesis. Proc Natl Acad Sci USA, 84: 2995-2998, 1987

(30) Galis ZS, Sukhova GK, Lark MW and Libby P: Increased expression of matrix metalloproteinases and matrix degrading activity in vulnerable regions of human atherosclerotic plaques. J Clin Invest, 94: 2493-2503, 1994

(31) Galis ZS, Sukhova GK, Kranzhofer R, Clark S and Libby P: Macrophage foam cells from experimental atheroma constitutively produce matrix-degrading proteinases. Proc Natl Acad Sci USA, 92: 402-406, 1995

(32) Matsumoto S, Kobayashi T, Katoh M, Saito S, Ikeda Y, Kobori M, Masuho $\mathrm{Y}$ and Watanabe T: Expression and localization of matrix metalloproteinase-12 in the aorta of cholesterol-fed rabbits: relationship to lesion development. Am J Pathol, 153: 109-119, 1998

(33) Wu L, Fan J, Matsumoto S and Watanabe T: Induction and regulation of matrix metalloproteinase-12 by cytokines and CD40 signaling in monocyte/macrophages. Biochem Biophys Res Commun, 269: 808-815, 2000

(34) Schonbeck U and Libby P: CD40 Signaling and Plaque Instability. Circ Res, 89: 1092-1103, 2001

(35) Steinberg D. Atherogenesis in perspective: Hypercholesterolemia and inflammation as partners in crime. Nat Med, 8: 1211-1217, 2002

(36) Fan J, Unoki H, Iwasa S and Watanabe T: Role of endothelin-1 in atherosclerosis. Ann NY Acad Sci, 902: 84-94, 2000

(37) Iwasa S, Fan J, Miyauchi T and Watanabe T: Blockade of endothelin receptors reduces diet-induced hypercholesterolemia and atherosclerosis in apolipoprotein E-deficient mice. Pathobiology, 69: $1-10,2001$

(38) Ichikawa $T$, Unoki $H$, Sun $H$, Shimoyamada $H$, Marcovina S, Shikama $\mathrm{H}$, Watanabe $\mathrm{T}$ and Fan $\mathrm{J}$ : Lipoprotein (a) promotes smooth muscle cell proliferation and dedifferentiation in atherosclerotic lesions of human apo (a) transgenic rabbits. Am J Pathol, 160: 227-236, 2002

(39) Sun H, Unoki H, Wang X, Liang J, Ichikawa T, Arai Y, Shiomi M, Marcovina SM, Watanabe $T$ and Fan $\mathrm{J}$ : Lipoprotein (a) enhances advanced atherosclerosis and vascular calcification in WHHL transgenic rabbits expressing human apolipoprotein (a). J Biol Chem, 277: 47486-47492, 2002

(40) Navab M, Berliner JA, Subbanagounder G, Hama S, Lusis AJ, Castellani LW, Reddy S, Shih D, Shi W, Watson $A D$, Van Lenten $B J$, Vora $D$ and Fogelman AM: HDL and the inflammatory response induced by LDL-derived oxidized phospholipids. Arterioscler Thromb Vasc Biol, 21: 481-488, 2001

(41) Nagy L, Tontonoz P, Alvarez JG, Chen H and Evans $\mathrm{RM}$ : Oxidized LDL regulates macrophage gene expression through ligand activation of PPAR gamma. Cell, 93: 229-240, 1998

(42) Tontonoz P, Nagy L, Alvarez JG, Thomazy VA and Evans RM: PPAR gamma promotes monocyte/macrophage differentiation and uptake of oxidized LDL. Cell, 93: 241-252, 1998

(43) Ricote M, Huang J, Fajas L, Li A, Welch J, Najib J, Witztum JL, Auwerx J, Palinski W and Glass CK: Expression of the peroxisome proliferator-activated receptor gamma (PPARgamma) in human atheroscle- 
rosis and regulation in macrophages by colony stimulating factors and oxidized low density lipoprotein. Proc Natl Acad Sci USA, 95: 7614-7619, 1998

(44) Duval C, Chinetti G, Trottein F, Fruchart J and Staels $\mathrm{B}$ : The role of PPARs in atherosclerosis. Trends Mol
Med, 8: 422, 2002

(45) Li A. C. and Glass C. K: The macrophage foam cell as a target for therapeutic intervention. Nat Med, 8: 1235-1242, 2002 